\title{
Effect of Strain Hardening on Elastic-Plastic Contact of a Deformable Sphere against a Rigid Flat under Full Stick Contact Condition
}

\author{
Biplab Chatterjee and Prasanta Sahoo \\ Department of Mechanical Engineering, Jadavpur University, Kolkata 700032, India \\ Correspondence should be addressed to Prasanta Sahoo, psjume@gmail.com
}

Received 7 June 2011; Revised 25 October 2011; Accepted 27 November 2011

Academic Editor: Kambiz Farhang

Copyright $\odot 2012$ B. Chatterjee and P. Sahoo. This is an open access article distributed under the Creative Commons Attribution License, which permits unrestricted use, distribution, and reproduction in any medium, provided the original work is properly cited.

\begin{abstract}
The present study considers the effect of strain hardening on elastic-plastic contact of a deformable sphere with a rigid flat under full stick contact condition using commercial finite element software ANSYS. Different values of tangent modulus are considered to study the effect of strain hardening. It is found that under a full stick contact condition, strain hardening greatly influences the contact parameters. Comparison has also been made between perfect slip and full stick contact conditions. It is observed that the contact conditions have negligible effect on contact parameters. Studies on isotropic and kinematic hardening models reveal that the material with isotropic hardening has the higher load carrying capacity than that of kinematic hardening particularly for higher strain hardening.
\end{abstract}

\section{Introduction}

Surface interactions are dependent on the contacting materials and the shape of the contacting surfaces. The shape of the surface of an engineering material is a function of both its production process and the nature of the parent material. When studied carefully on a very fine scale, all solid surfaces are found to be rough. So when two such surfaces are pressed together under loading, only the peaks or the asperities of the surface are in contact and the real area of contact is only a fraction of the apparent area of contact. In such conditions the pressure in those contact spots are high. Accurate calculation of contact area and contact load are of immense importance in the field of tribology and lead to an improved understanding of friction, wear, and thermal and electrical conductance between surfaces. However, it is a difficult task as rough surfaces consist of asperities having different radius and height. The problem is simplified when Hertz [1] provides the contact analysis of two elastic solids with geometries defined by quadratic surfaces. From then, the assumption of surfaces having asperities of spherical shape is adopted to simplify the contact problems. Greenwood and Williamson [2] used the Hertz theory and proposed an asperity-based elastic model where asperity heights follow a Gaussian distribution. Hertz assumed frictionless surfaces and his theory is restricted for perfectly elastic solids. Later on, researchers have attempted to investigate the effect of material properties beyond the Hertz restriction and the elastic plastic contact of a sphere with a flat became a fundamental problem in contact mechanics. The plastic model introduced by Abbot and Firestone [3], neglects volume conservation of the plastically deformed sphere. One of the first model of elastic-plastic contact was proposed by Chang et al. (CEB model) [4], where the sphere remains in elastic contact until a critical interference is reached, above which the volume conservation of the sphere tip is imposed. The CEB model suffers from a discontinuity in the contact load as well as in the first derivative of both the contact load and the contact area at the transition from elastic to elastic-plastic region. Later Evseev et al. [5], Chang [6], and Zhao et al. [7] have made attempts to improve the elastic-plastic contact model. An introduction of friction at the interface of contact had enabled the Hertz theory to be extended in a realistic manner. Timoshenko and 
Goodier [8] stated that the results of normal loading under friction differ from the frictionless Hertzian contact problem. However, contact of spheres with the same elastic constants yields identical tangential displacements, which eliminates the possibility of interfacial slip and the Hertz theory is applicable in certain cases of frictional contact also [9]. Hence normal contact of two spheres with the same material properties exhibits the same results under full stick contact and perfect slip contact condition. This idea is used in modeling spherical contact under combined normal and tangential loading by several researchers (Mindlin [10], Bryant and Keer [11], Hamilton [12], Chang et al. [13]). These authors [10-13] assumed perfect slip contact during normal loading and used friction for tangential loading. Several researchers (Vijaywargiya and Green [14], Boucly et al. [15], Jackson et al. [16]) have also considered the elastic-plastic contact of interfering cylinders/spheres. Goodman [17] first provided the analytical solution of two dissimilar elastic spheres in normal contact under full stick (infinite friction) condition. Spence [18] solved simultaneously the dual integral equations for shear stresses and pressure distribution over the contact area and calculated the total compressive load under the full stick condition. Spence [19] extended his previous work to adhesive contact using a certain value of the friction coefficient. He found that the extent of slip region remains the same for the same elastic constants and friction coefficient.

The studies of contact mechanics of a deformable sphere and a rigid flat revealed the effect of contact conditions and material properties on the deformation and the interfacial parameters like contact load and contact area. However, accurate solutions could not be done until the finite element method was used to solve the problems. Commercial finite element software has the capability to calculate accurately the contact parameters like contact load, contact area, and pressure, and so forth removing some of the assumptions made in the earlier theories regarding asperity interaction and small deformations [20]. Kogut and Etsion [21] (KE Model) first provided an accurate result of elastic-plastic contact of a hemisphere and a rigid flat using commercial finite element software ANSYS to study the plastic zone under frictionless contact condition. They considered a wide range of material properties and sphere size and provided generalized empirical relations for contact area and contact force in terms of dimensionless contact interference for elastic, elastic-plastic, and fully plastic region. They also considered a variation of the tangent modulus up to $0.1 \mathrm{E}$ and found negligible effect on the contact parameters. A similar analysis has been done by Jackson and Green [22] (JG Model). The JG model observed the effect of the deformed geometry on the effective hardness and presented some empirical relations of contact area and contact load. Quicksall et al. [23] used finite element technique to model the elastic-plastic deformation of a hemisphere in contact with a rigid flat for various materials such as aluminum, bronze, copper, titanium, and malleable cast iron. They also studied the contact parameters for a generic material in which the elastic modulus and Poisson's ratio were independently varied keeping the yield strength constant. Shankar and Mayuram $[24,25]$ considered the effect of material properties during transition from elastic to elasticplastic region using the KE model. Recently, Malayalamurthi and Marappan [26] and Sahoo et al.[27, 28] concluded that the interfacial parameters like contact load, real contact area during loading are dependent on the material properties of the deformable sphere. So it can be seen that a wide range of literature is available for frictionless contact of a deformable sphere and a rigid flat. The ideal assumption of frictionless contact (perfect slip) may give an idea for interfacial interactions, but results differ from the realistic contact condition where friction is present. According to Johnson [29], "Friction can increase the total load required to produce a contact of given size by at most $5 \%$ compared with Hertz."

Two types of contact conditions are in general available in literature; perfect slip and full stick. In perfect slip, it is assumed that there is no tangential stress in the contact area. In full stick, the contacting points of the sphere and the flat, which are covered by the expanding contact zone, are prevented from further relative displacement [30]. Brizmer et al. [31] first analyzed the effect of full stick condition on the elasticity terminus of a spherical contact using finite element software ANSYS. Brizmer et al. [32] extended their study for the loading of an elastic plastic spherical contact both under full stick and perfect slip contact conditions. They found that the interfacial parameters are insensitive to the contact condition and material properties of the deformable sphere. However the contact loads and average contact pressure are slightly affected by Poisson's ratio for full stick contact condition. Zait et al. [33] performed the unloading of an elastic-plastic spherical contact under full stick contact condition. They found a substantial variation in load area curve during unloading under full stick contact condition compared to that of under perfect slip condition.

Kogut and Etsion [21] studied the effect of strain hardening with the tangential modulus up to $0.1 \mathrm{E}$ and concluded negligible effect of strain hardening for frictionless and nonadhesive contact. Sahoo et al. [27] used different tangent modulus (maximum 0.33E) to study the effect of strain hardening on contact parameters for frictionless and non-adhesive contact. They inferred that, with the increase in strain hardening the resistance to deformation of a material is increased and the material becomes capable of carrying higher amount of load in a smaller contact area. Kadin et al. [34] observed that secondary plastic flow is strongly affected by strain hardening and they suggested investigating the behavior with the kinematic material hardening for subsequent multiple loading-unloading. However the effect of strain hardening for the full stick contact condition is still not available in literature. Thus present investigation attempts to study the effect of strain hardening on contact parameters for full stick contact condition.

\section{Theoretical Background}

The contact of a deformable hemisphere and a rigid flat is shown in Figure 1 where the dashed and solid lines represent the situation before and after contact, respectively, of the sphere of radius $R$. The figure also shows the interference $(\omega)$ and contact radius (a) corresponding to a contact 


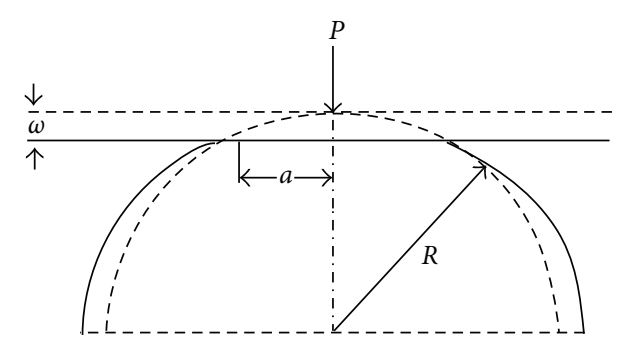

Figure 1: A deformable sphere pressed by a rigid flat.

load $(P)$. As discussed earlier the present study is concentrated on the full stick contact condition and compared with results [27] for perfect slip contact condition. Tabor [35] mentioned that full stick contact condition is more realistic than that of perfect slip contact condition due to the formation of an adhesive junction at the interface. Brizmer et al. [32] provided the empirical relations for critical interference and corresponding values for critical loads and critical contact area for perfect slip and full stick contact conditions. For full stick contact condition, the contact parameters are normalized using the expressions of Brizmer et al. [32] for critical interference $\left(\omega_{c}\right)$, critical load $\left(P_{c}\right)$, and critical contact area $\left(A_{c}\right)$ given by the following:

$$
\omega_{c}=\left(C_{v} \frac{\pi\left(1-v^{2}\right)}{2}\left(\frac{Y}{E}\right)\right)^{2} R\left(6.82 v-7.83\left(v^{2}+0.0586\right)\right),
$$

$P_{c}=\frac{\pi^{3} Y}{6} C_{v}^{3}\left(R\left(1-v^{2}\right)\left(\frac{Y}{E}\right)\right)^{2}\left(8.88 v-10.13\left(v^{2}+0.089\right)\right)$,

$$
A_{c}=\pi \omega_{c} R,
$$

where, $C_{v}=1.234+1.256 v$. The parameters $Y, E$, and $v$ are the yield stress, Young modulus, and Poisson's ratio of the sphere material, respectively, and $R$ is the radius of the sphere.

\section{Finite Element Model}

The contact of the deformable sphere with a rigid flat is modeled using finite element software (ANSYS 11). Due to the advantage of simulation of axisymmetric problems the model is reduced to a quarter circle with a straight line at its top as shown in Figure 2.

The quarter circle is divided into two different zones, for example, zone I and zone II. Zone I is within 0.1R distance from the sphere tip and zone II is the remaining region of the circle outside zone I. These two zones are significant according to their mesh density. The mesh density of zone I is high enough for the accurate calculation of the contact area of the sphere under deformation. Zone II has a coarser mesh as this zone is far away from the contact zone. The resulting mesh consists of 12986 of PLANE82 and 112 of CONTA172 elements. The rigid flat is modeled by a single, nonflexible two-node target surface element (TARGE169). The nodes lying on the axis of symmetry of the hemisphere are restricted to move only in the radial direction. Also the nodes in the bottom of the hemisphere are restricted in the axial direction due to symmetry. The sphere size is used for this analysis is $R=1 \mu \mathrm{m}$. The material properties used here are Young's Modulus $(E)=70 \mathrm{GPa}$, Poisson's Ratio $(\nu)=0.3$, and Yield stress $\left(\sigma_{y}\right)=100 \mathrm{MPa}$. For full stick contact condition, an infinite friction condition is adopted. The ANSYS solution type is chosen as large deformation static analysis. The bilinear isotropic hardening (BISO) option in the ANSYS program is chosen to account the elastic-plastic material response for the single-asperity model. Results are compared with kinematic material hardening as well. Here displacement is applied on the target surface and the force on the hemisphere is found from the reaction solution. As this is an axisymmetric analysis the force is calculated on a full-scale basis. In the present analysis mesh configuration is validated by iteratively increasing the mesh density. The mesh density is doubled until the contact force and contact area is differed by less than $1 \%$ between the iterations. In addition to mesh convergence, the model also compares well with the Hertz elastic solution at interferences below the critical interference for perfect slip contact condition. This work uses the Lagrangian multiplier method. The tolerance of the current work is set to $1 \%$ of the element width. Computations took about 15 minutes for getting solutions up to yield inception and an hour for large deformation in a $1.6 \mathrm{GHz}$. PC.

\section{Results and Discussion}

Strain hardening is an increase in the strength and hardness of the metal due to a mechanical deformation in the microstructure of the metal. This is caused by the cold working of the metal. Strain hardening is expressed in terms of tangent modulus $\left(E_{t}\right)$ which is the slope of the stress-strain curve. Below the proportional limit the tangent modulus is the same as the Young's modulus. Above the proportional limit the tangent modulus varies with the strain unless bilinear hardening is used. The tangent modulus is useful in describing the behavior of materials that have been stressed beyond the elastic region. When a material is plastically deformed there is no longer a linear relationship between the stress and strain as there is for elastic deformation. In elastic perfectly plastic cases, the tangent modulus tends to be zero. Very few material, exhibit elastic perfectly plastic behaviors, generally all the materials follow the multi-linear behavior with some tangent modulus. This multilinear behavior can be assumed as bilinear behavior for analysis purpose in elastic-plastic cases. In this analysis a bilinear material property, as shown in Figure 3, is assumed for the deformable hemisphere.

To study the strain hardening effect, different values of tangent modulus $E_{t}$ are considered. The tangent modulus $E_{t}$ is varied according to a parameter, which is known as hardening parameter $(H)$ and defined as $H=E_{t} /\left(E-E_{t}\right)$. The value of $H$ is taken in the range $0 \leq H \leq 0.5$ as most of the practical materials falls in this range [36]. The value of $H$ equals to zero indicates elastic perfectly plastic 


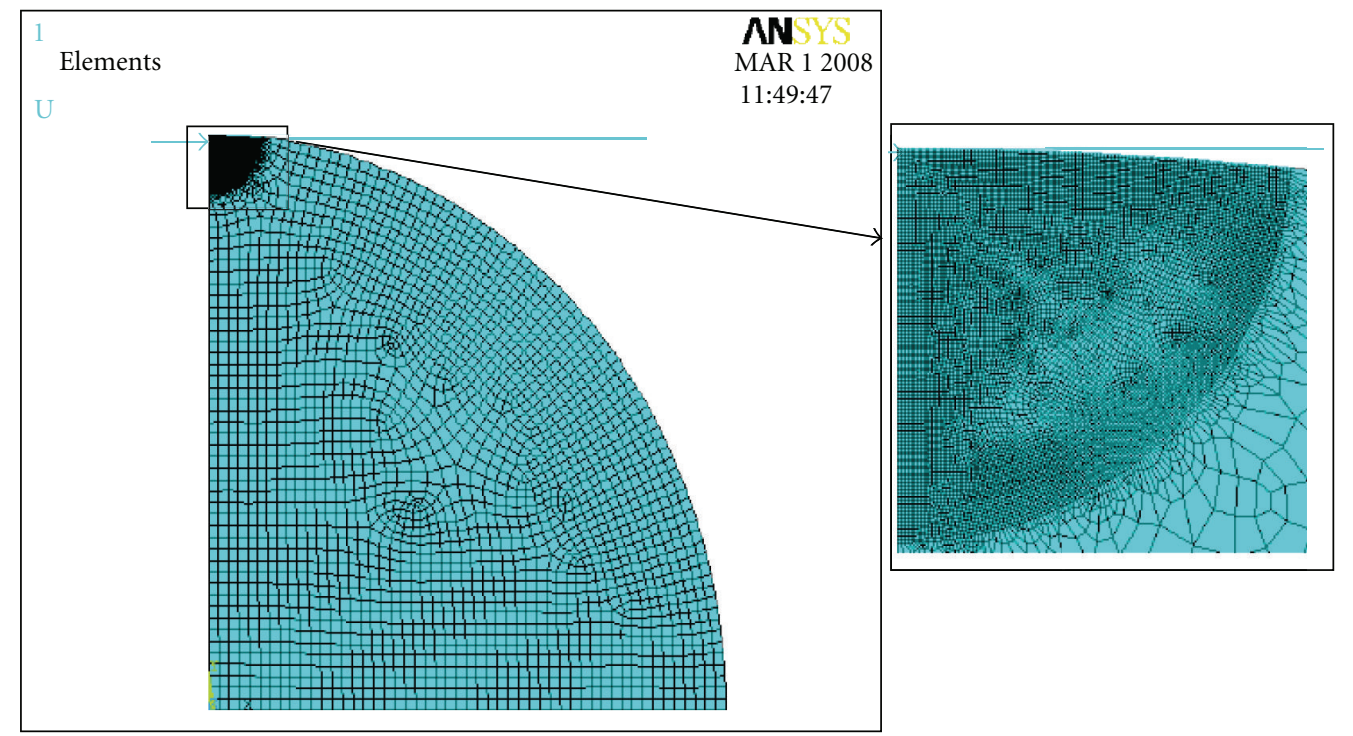

FIgURE 2: Meshed model of the hemispherical contact.

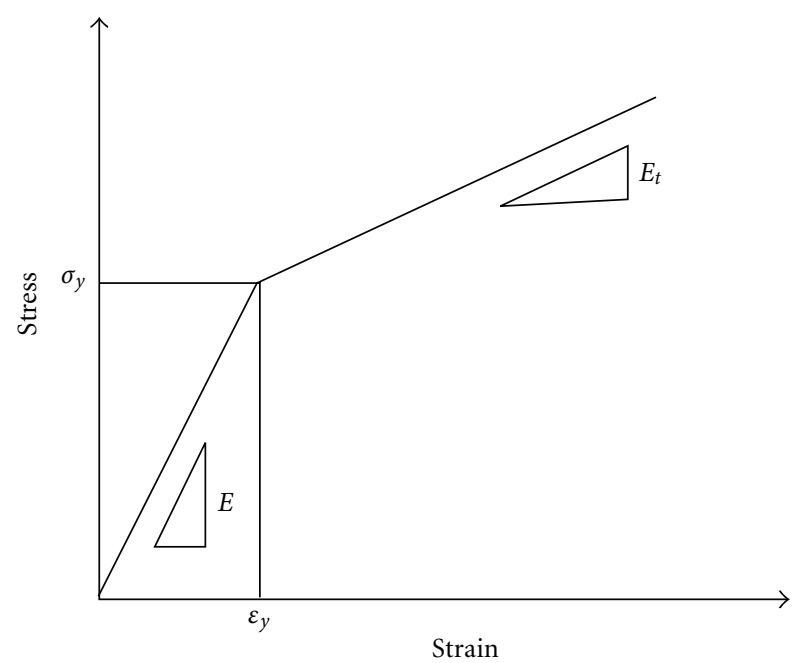

FIgURE 3: Stress-strain diagram for a material having bilinear isotropic properties.

material behavior, which is an idealized material behavior. The hardening parameters used for this analysis and their corresponding $E_{t}$ values are shown in Table 1 . The wide range of values of tangent modulus is taken to make a fair idea of the effect of strain hardening in single-asperity contact analysis with the other material properties being constant.

Figure 4 presents the comparison of dimensionless contact load as a function of dimensionless interference for various models. KE model [21] and Sahoo et al. [27] consider perfect slip contact condition with elastic perfectly plastic material, whereas Brizmer et al. [32] analyze full stick contact condition assuming the material of the sphere as elastic linear isotropic hardening with a tangent modulus of $2 \%$ of the Young modulus. The maximum variation of dimensionless
TABLE 1: Different $H$ and $E_{t}$ values used for the study of strain hardening effect.

\begin{tabular}{lcc}
\hline$H$ & $E_{t}$ in $\% E$ & $E_{t}(\mathrm{GPa})$ \\
\hline 0 & 0.0 & 0.0 \\
0.1 & 9.0 & 6.3 \\
0.2 & 16.7 & 11.7 \\
0.3 & 23.0 & 16.1 \\
0.4 & 28.6 & 20.0 \\
0.5 & 33.0 & 23.1 \\
\hline
\end{tabular}

contact load between KE model and Sahoo et al. is 3\%. It may be noted here that Kogut and Etsion considered a large number of sphere radii in the range of $0.1 \leq R \leq 10(\mathrm{~mm})$ as well as a large variation of material properties in the range $100 \leq\left(E / \sigma_{y}\right) \leq 1000$, and they also found differences in their results up to $3 \%$. The present results with elastic perfectly plastic material under full stick contact condition agree well with the findings of Brizmer et al.

Figure 5 shows the variation of contact load at different interferences for materials having different values of tangent modulus under full stick contact condition. The plot shows a nonlinear behavior between the load and interference as the results are in the elastic-plastic and fully plastic region. The plot shows that up to a certain value of nondimensional interference $\left(\omega / \omega_{c}=10\right)$ the effect of strain hardening on contact parameters is negligible as in the case of perfect slip contact condition. The variation of hardening parameters shows that for a small hardening parameter $(H=0.1)$, the dimensionless contact load increases by $3-21 \%$ compared to the results of elastic perfectly plastic case with in the elasticplastic region, that is, $10 \omega_{c} \leq \omega \leq 100 \omega_{c}$. While for the large hardening parameter $(H=0.5)$, the increase in dimensionless contact load is in the range $11-57 \%$ compared to that of elastic perfectly plastic case in the elastic-plastic region. In 


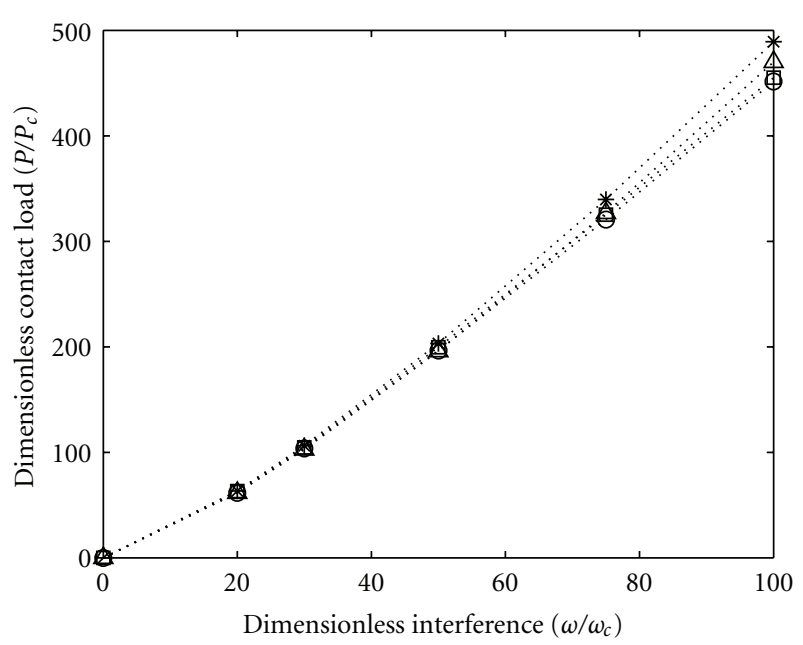

. o. Sahoo et al. slip contact $\cdots * \cdot$ Brizmer et al. stick contact $\Delta$. KE model slip contact ..... Present work stick contact

Figure 4: Dimensionless contact load versus dimensionless interference for various models.

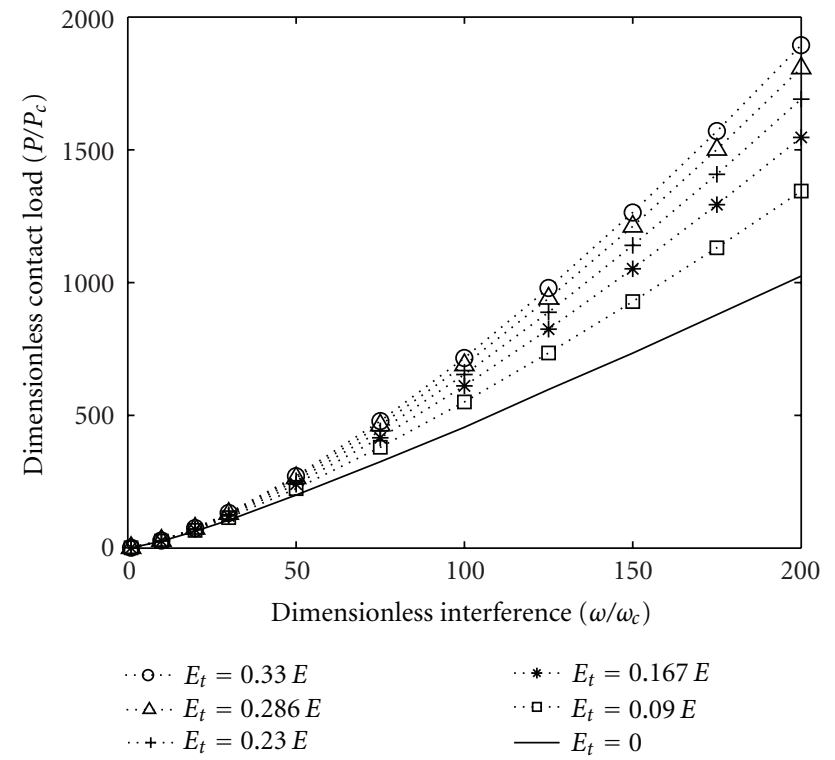

Figure 5: Dimensionless contact load versus dimensionless interference at stick contact condition.

fully plastic region these variations are significantly high and increase monotonically with the increase in interference.

Figure 6 represents the plot of dimensionless contact area with dimensionless interference for materials having different values of tangent modulus under full stick contact condition. For perfect slip contact condition the compressible sphere material tends to displace inward during loading up to $\omega / \omega_{c}=22$ (Jackson and Green [22]). It is revealed from the finite element analysis that at $\omega / \omega_{c}=20$, the dimensionless contact area for full stick contact condition is $3-12 \%$ higher than the corresponding dimensionless contact area under perfect slip contact condition. This behavior is expected as

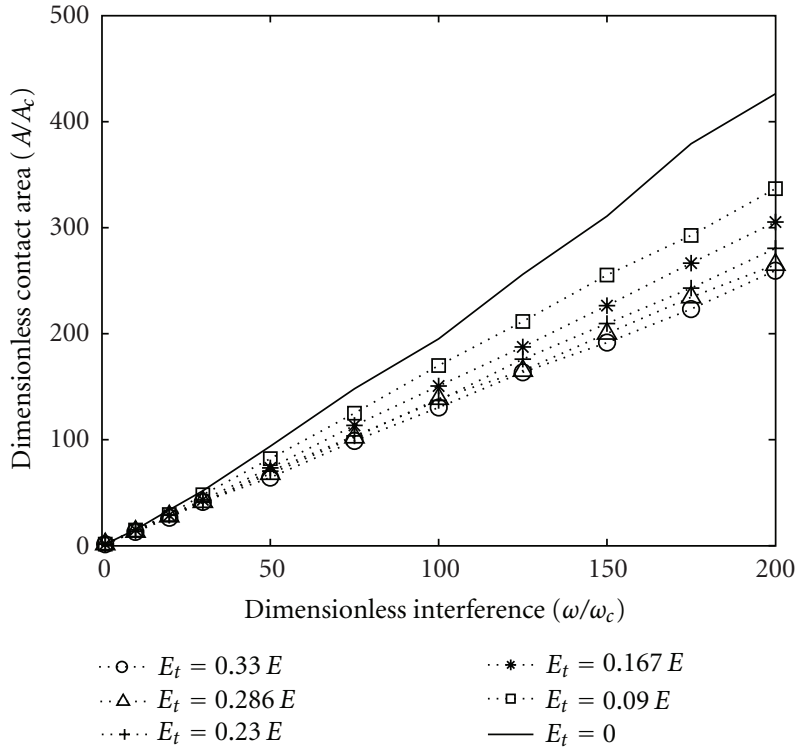

Figure 6: Dimensionless contact area versus dimensionless interference for stick contact condition.

the sphere material could not be displaced inward owing to the stick imposed by the rigid surface. The variation of hardening parameter shows that for a small hardening parameter $(H=0.1)$, the dimensionless contact area decreases by $3-$ $13 \%$ compared to the elastic perfectly plastic case with in the elastic-plastic region, that is, $10 \omega_{c} \leq \omega \leq 100 \omega_{c}$. While for large hardening parameter $(H=0.5)$, the decrease in dimensionless contact area is in the range of $14-33 \%$ compared to the elastic perfectly plastic case in the elastic-plastic region.

Figure 7 shows the variation of contact area at different applied loads for materials having different values of tangent modulus. The figure shows a nonlinear behavior between the contact area and contact force. Here it is observed that the contact area is less at a particular load for a material having a higher tangent modulus value than that of a material having a lower tangent modulus. This indicates that with the increase in the effect of strain hardening, the material can support the same applied load in a smaller contact area.

The effect of kinematic bilinear hardening is also studied here. Figure 8 describes the difference between isotropic and kinematic bilinear hardening by describing the development of the yield surface with progressive yielding. In isotropic or work hardening, the yield surface is uniformly spread out from the center while in kinematic hardening the yield surface translates in stress space with constant size. Figure 9 presents the dimensionless contact load as a function of dimensionless interference for both the kinematic and isotropic bilinear hardening models. Zait et al. [37] suggested that the effect of hardening model would be most noticeable for large stress amplitudes during a single loading-unloading cycle. Thus here a comparison is made to study the effect of hardening model for three hardening parameter $H=$ $0,0.3$ and 0.5 up to 200 times of critical interference during loading. It is revealed from the figure that for hardening 


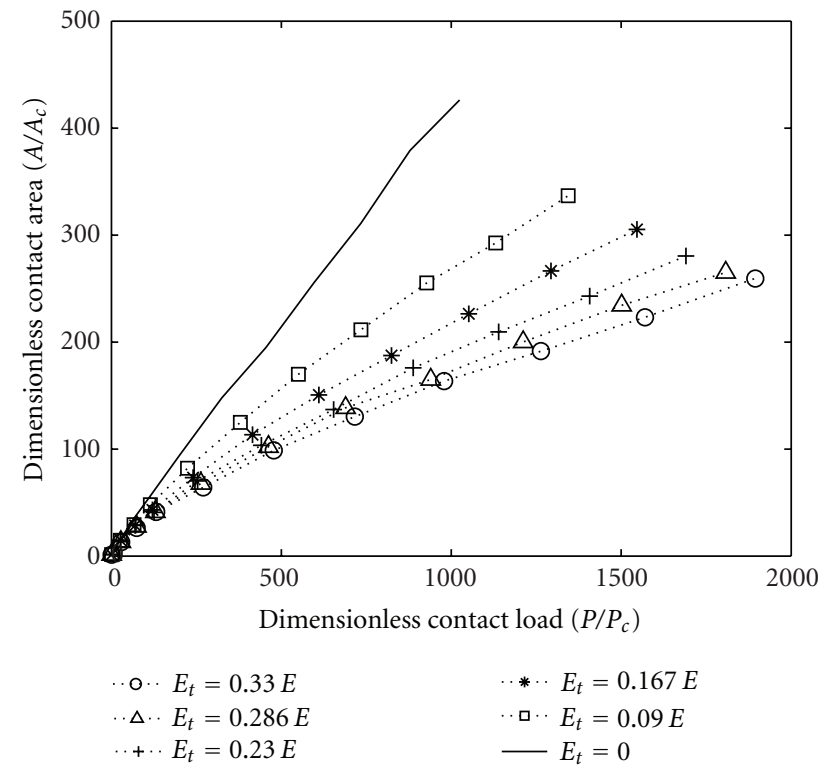

FIGURE 7: Dimensionless contact load versus dimensionless contact area for stick contact condition.

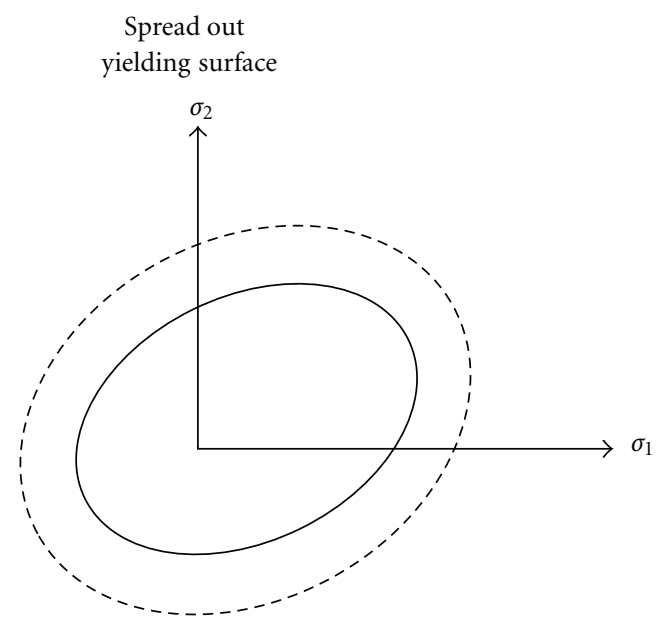

(a)

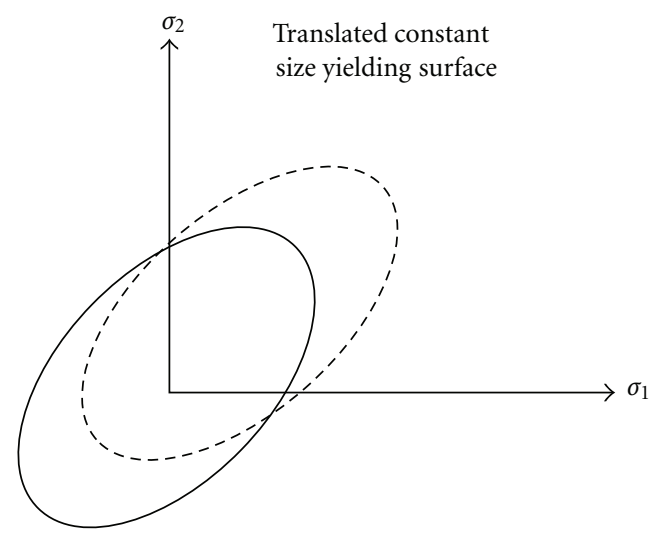

(b)

FIGURe 8: Isotropic (a) and kinematic (b) hardening models for two-dimensional stress field.

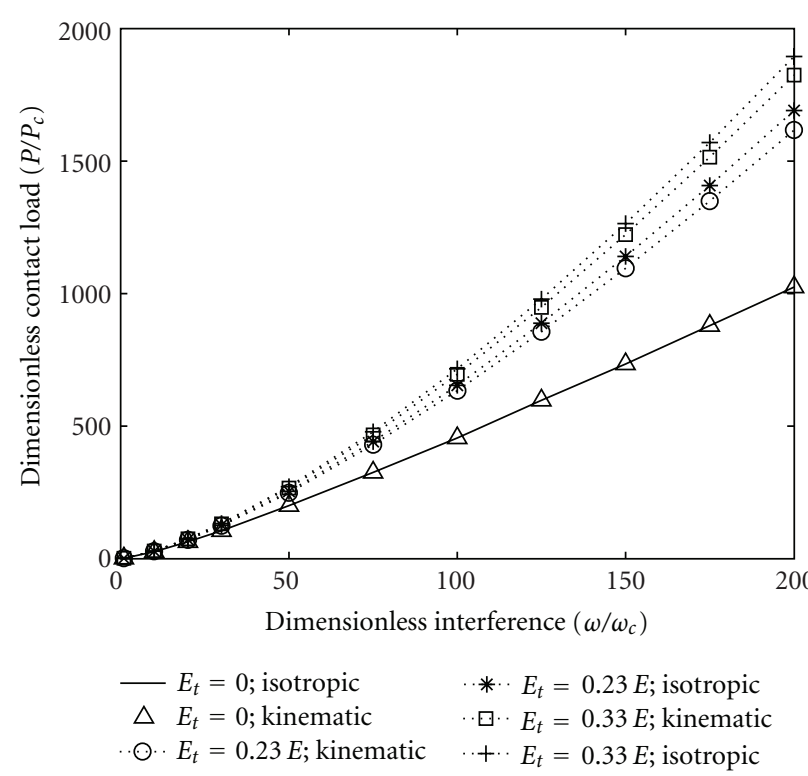

FIGURE 9: Dimensionless contact load against dimensionless interference for kinematic and isotropic material hardening.

parameter of 0 , that is, for elastic perfectly plastic material the dimensionless contact load for both the hardening models is exactly same even for larger interferences. Zait et al. [37] did not find any significant variation for both the hardening models. They used $2 \%$ tangent modulus $\left(E_{t} / E=2 \%\right)$ and studied up to $\omega / \omega_{c}=150$. For hardening parameter of 0.3 and 0.5 that is; for tangent modulus of $23 \%$ and $33 \%$ of Young's modulus $E$, it is found from the present simulation that the dimensionless contact load is higher for isotropic hardening model than that of kinematic hardening model at larger interferences. For hardening parameter of 0.3 and 0.5 , the dimensionless contact loads are higher by $1.5-4.6 \%$ and $1.4-3.9 \%$, respectively, for isotropic hardening model than that of kinematic hardening model.

Figure 10 shows the variation of dimensionless contact area with the variation of dimensionless interference for both the kinematic and isotropic hardening models. Here also the dimensionless contact area is same for both the hardening models in case of elastic perfectly plastic material. For hardening parameter of 0.3 and 0.5 , the dimensionless contact area is larger in kinematic hardening model than that for isotropic hardening model. It is revealed from the figure that at hardening parameter of 0.3 and 0.5 ; the dimensionless contact areas are higher by $0.4-2.9 \%$ and $0.15-1.8 \%$, respectively, in kinematic hardening model than that of isotropic hardening model. Figure 11 shows the plots of dimensionless contact area versus dimensionless contact load for both the kinematic and isotropic hardening models. Here it is found that with isotropic hardening model, material can support a particular load with a smaller contact area compared to kinematic hardening for higher rate of hardening. The experimental results of Ovcharenko et al. [38] for full stick contact conditions have also been incorporated in Figure 11. It may be noted that the present theoretical results compare 


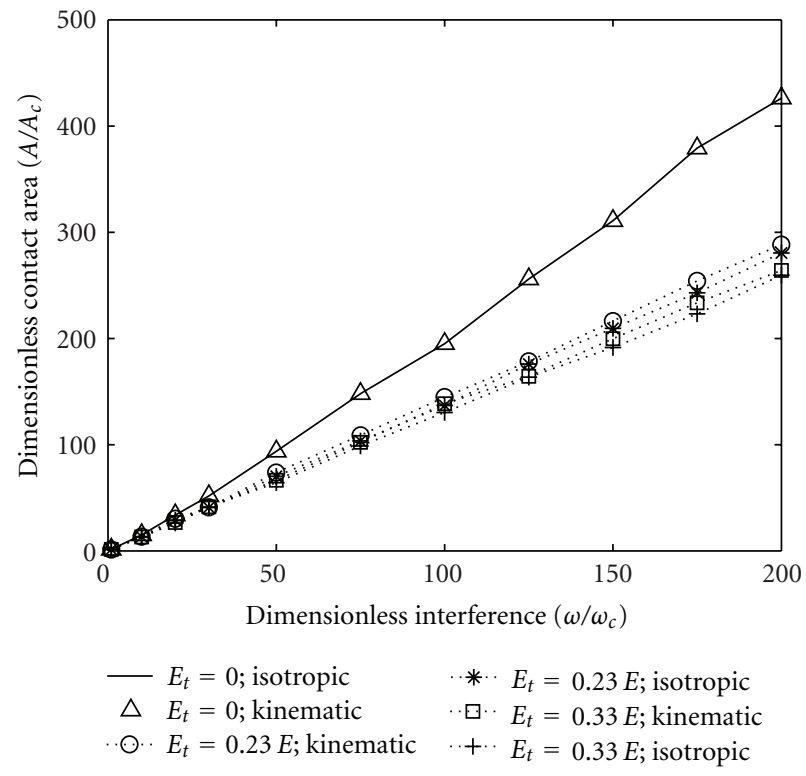

FIGURE 10: Dimensionless contact area against dimensionless interference for kinematic and isotropic material hardening.

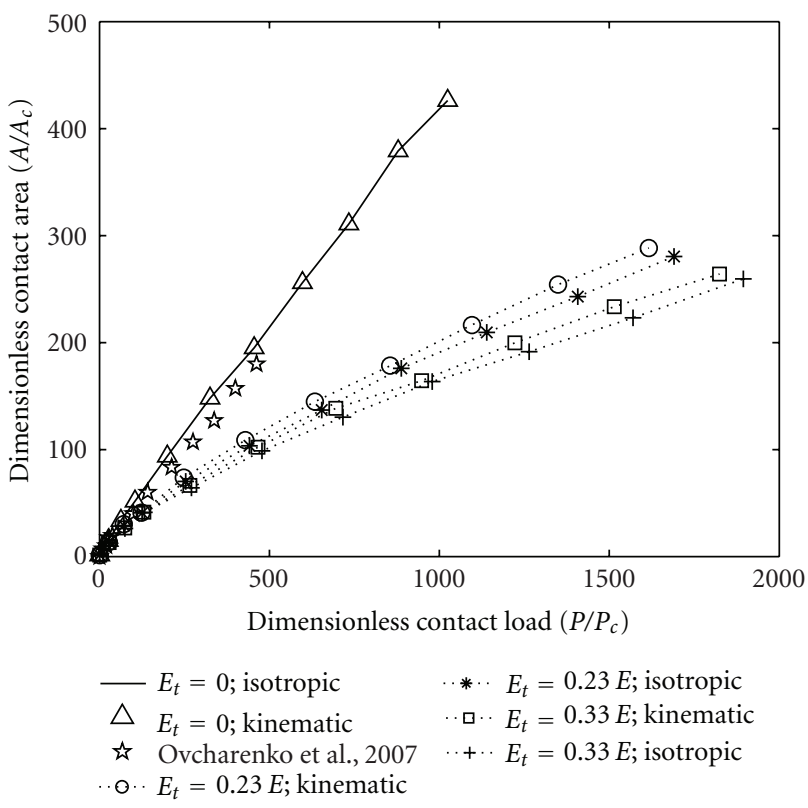

Figure 11: Dimensionless contact load versus dimensionless contact area for kinematic and isotropic material hardening.

well with the experimental results. It may also be noted here that any significant difference between the two hardening models is observed only when the loading is reversed [39].

\section{Conclusion}

The effect of strain hardening on contact parameters under the full stick contact condition for both the isotropic and kinematic hardening models is investigated. It is found that, a generalized behavior cannot be applicable under full stick contact condition for all kind of materials as the effect of strain hardening greatly influenced the contact parameters. The dimensionless contact load increases with the increase in strain hardening while the dimensionless contact area decreases. It is also observed that the contact conditions have little effect on the interfacial parameters like contact load and contact area. With the increase in strain hardening, the resistance to deformation of a material increases and the material becomes capable of carrying a higher amount of load in a smaller contact area. The material with isotropic hardening can support higher amount of load with same contact area compared to that for material having kinematic hardening behavior.

\section{References}

[1] H. Hertz, "Über die Berührung fester elastischer Köper," Journal für die Reine und Angewandte Mathematik, vol. 92, pp. 156-171, 1882.

[2] J. A. Greenwood and J. B. P. Williamson, "Contact of nominally flat surfaces," Proceedings of the Royal Society of London. Series A, vol. 295, pp. 300-319, 1966.

[3] E. J. Abbott and F. A. Firestone, "Specifying surface qualitya method based on accurate measurement and comparison," ASME Journal of Mechanical Engineering, vol. 55, pp. 569-572, 1933.

[4] W. R. Chang, I. Etsion, and D. B. Bogy, "An elastic-plastic model for the contact of rough surfaces," ASME Journal of Tribology, vol. 109, no. 2, pp. 257-263, 1987.

[5] D. G. Evseev, B. M. Medvedev, and G. G. Grigoriyan, "Modification of the elastic-plastic model for the contact of rough surfaces," Wear, vol. 150, no. 1-2, pp. 79-88, 1991.

[6] W. R. Chang, "An elastic-plastic contact model for a rough surface with an ion-plated soft metallic coating," Wear, vol. 212, no. 2, pp. 229-237, 1997.

[7] Y. Zhao, D. M. Maietta, and L. Chang, "An asperity microcontact model incorporating the transition from elastic deformation to fully plastic flow," ASME Journal of Tribology, vol. 122, no. 1, pp. 86-93, 2000.

[8] S. P. Timoshenko and J. N. Goodier, Theory of Elasticity, McGraw-Hill, New York, NY, USA, 3rd edition, 1970.

[9] K. L. Johnson, "One hundred years of Hertz contact," Proceedings of the Institution of Mechanical Engineers, vol. 196, no. 1, pp. 363-378, 1982.

[10] R. D. Mindlin, "Compliance of elastic bodies in contact," ASME Journal of Applied Mechanics, vol. 16, pp. 259-268, 1949.

[11] M. D. Bryant and L. M. Keer, "Rough contact between elastically and geometrically identical curved bodies," ASME Journal of Applied Mechanics, vol. 49, no. 2, pp. 345-352, 1982.

[12] G. M. Hamilton, "Explicit equations for the stresses beneath a sliding spherical contact," Proceedings of the Institution of Mechanical Engineers, vol. 197, pp. 53-59, 1983.

[13] W. R. Chang, I. Etsion, and D. B. Bogy, "Static friction coefficient and model for metallic rough surfaces," Journal of Tribology, vol. 110, no. 1, pp. 57-63, 1988.

[14] R. Vijaywargiya and I. Green, "A finite element study of the deformations, forces, stress formations, and energy losses in sliding cylindrical contacts," International Journal of NonLinear Mechanics, vol. 42, no. 7, pp. 914-927, 2007.

[15] V. Boucly, D. Nelias, and I. Green, "Modeling of the rolling and sliding contact between two asperities," Journal of Tribology, vol. 129, no. 2, pp. 235-245, 2007. 
[16] R. L. Jackson, R. S. Duvvuru, H. Meghani, and M. Mahajan, "An analysis of elasto-plastic sliding spherical asperity interaction," Wear, vol. 262, no. 1-2, pp. 210-219, 2007.

[17] L. E. Goodman, "Contact stress analysis of normally loaded rough spheres," ASME Journal of Applied Mechanics, vol. 29, no. 3, pp. 515-522, 1962.

[18] D. A. Spence, "Self-similar solutions to adhesive contact problems with incremental loading," Proceedings of the Royal Society of London A, vol. 305, pp. 55-80, 1968.

[19] D. A. Spence, "The Hertz contact problem with finite friction," Journal of Elasticity, vol. 5, no. 3-4, pp. 297-319, 1975.

[20] P. Sahoo, D. Adhikary, and K. Saha, "Finite element based elastic-plastic contact of fractal surfaces considering strain hardening," Journal of Tribology and Surface Engineering, vol. 1, no. 1-2, pp. 39-56, 2010.

[21] L. Kogut and I. Etsion, "Elastic-plastic contact analysis of a sphere and a rigid flat," ASME Journal of Applied Mechanics, vol. 69, no. 5, pp. 657-662, 2002.

[22] R. L. Jackson and I. Green, "A finite element study of elastoplastic hemispherical contact against a rigid flat," ASME Journal of Tribology, vol. 46, no. 2, pp. 383-390, 2003.

[23] J. J. Quicksall, R. L. Jackson, and I. Green, "Elasto-plastic hemispherical contact models for various mechanical properties," Proceedings of the Institution of Mechanical Engineers, Part J: Journal of Engineering Tribology, vol. 218, pp. 313-322, 2004.

[24] S. Shankar and M. M. Mayuram, "A finite element based study on the elastic-plastic transition behavior in a hemisphere in contact with a rigid flat," Journal of Tribology, vol. 130, no. 4, Article ID 044502, 6 pages, 2008.

[25] S. Shankar and M. M. Mayuram, "Effect of strain hardening in elastic-plastic transition behavior in a hemisphere in contact with a rigid flat," International Journal of Solids and Structures, vol. 45, no. 10, pp. 3009-3020, 2008.

[26] R. Malayalamurthi and R. Marappan, "Elastic-plastic contact behavior of a sphere loaded against a rigid flat," Mechanics of Advanced Materials and Structures, vol. 15, no. 5, pp. 364-370, 2008.

[27] P. Sahoo, B. Chatterjee, and D. Adhikary, "Finite element based elastic-plastic contact behavior of a sphere against a rigid flat- effect of strain hardening," International Journal of Engineering, Science and Technology, vol. 2, no. 1, pp. 1-6, 2010.

[28] P. Sahoo and B. Chatterjee, "A finite element study of elasticplastic hemispherical contact behavior against a rigid flat under varying modulus of elasticity and sphere radius," Engineering, vol. 2, no. 4, pp. 205-211, 2010.

[29] K. L. Johnson, Contact Mechanics, Cambridge University Press, Cambridge, Mass, USA, 1985.

[30] K. L. Johnson, J. J. O' Conner, and A. C. and Woodward, “The effect of the indenter elasticity on the Hertzian fracture of brittle materials," Proceedings of the Royal Society of London A, vol. 334, no. 1596, pp. 95-117, 1973.

[31] V. Brizmer, Y. Kligerman, and I. Etsion, "The effect of contact conditions and material properties on the elasticity terminus of a spherical contact," International Journal of Solids and Structures, vol. 43, no. 18-19, pp. 5736-5749, 2006.

[32] V. Brizmer, Y. Kligerman, and I. Etsion, "The effect of contact conditions and material properties on elastic-plastic spherical contact," Journal of Mechanics of Materials and Structures, vol. 1, no. 5, pp. 865-879, 2006.

[33] Y. Zait, Y. Kligerman, and I. Etsion, "Unloading of an elastic-plastic spherical contact under stick contact condition," International Journal of Solids and Structures, vol. 47, no. 7-8, pp. 990-997, 2010.

[34] Y. Kadin, Y. Kligerman, and I. Etsion, "Multiple loading-unloading of an elastic-plastic spherical contact," International
Journal of Solids and Structures, vol. 43, no. 22-23, pp. 71197127, 2006.

[35] D. Tabor, "Junction growth in metallic friction: the role of combined stresses and surface contamination," Proceedings of the Royal Society of London. Series A, vol. 251, no. 1266, pp. 378-393, 1959.

[36] F. Wang and L. M. Keer, "Numerical simulation for three dimensional elastic-plastic contact with hardening behavior," Journal of Tribology, vol. 127, no. 3, pp. 494-502, 2005.

[37] Y. Zait, V. Zolotarevsky, Y. Klingerman, and I. Etsion, "Multiple normal loading-unloading cycles of a spherical contact under stick contact condition," Journal of Tribology, vol. 132, no. 4, Article ID 041401, 7 pages, 2010.

[38] A. Ovcharenko, G. Halperin, G. Verberne, and I. Etsion, "In situ investigation of the contact area in elastic-plastic spherical contact during loading-unloading," Tribology Letters, vol. 25, no. 2, pp. 153-160, 2007.

[39] V. Zolotarevskiy, Y. Kligerman, and I. Etsion, "Elastic-plastic spherical contact under cyclic tangential loading in presliding," Wear, vol. 270, no. 11-12, pp. 888-894, 2011. 

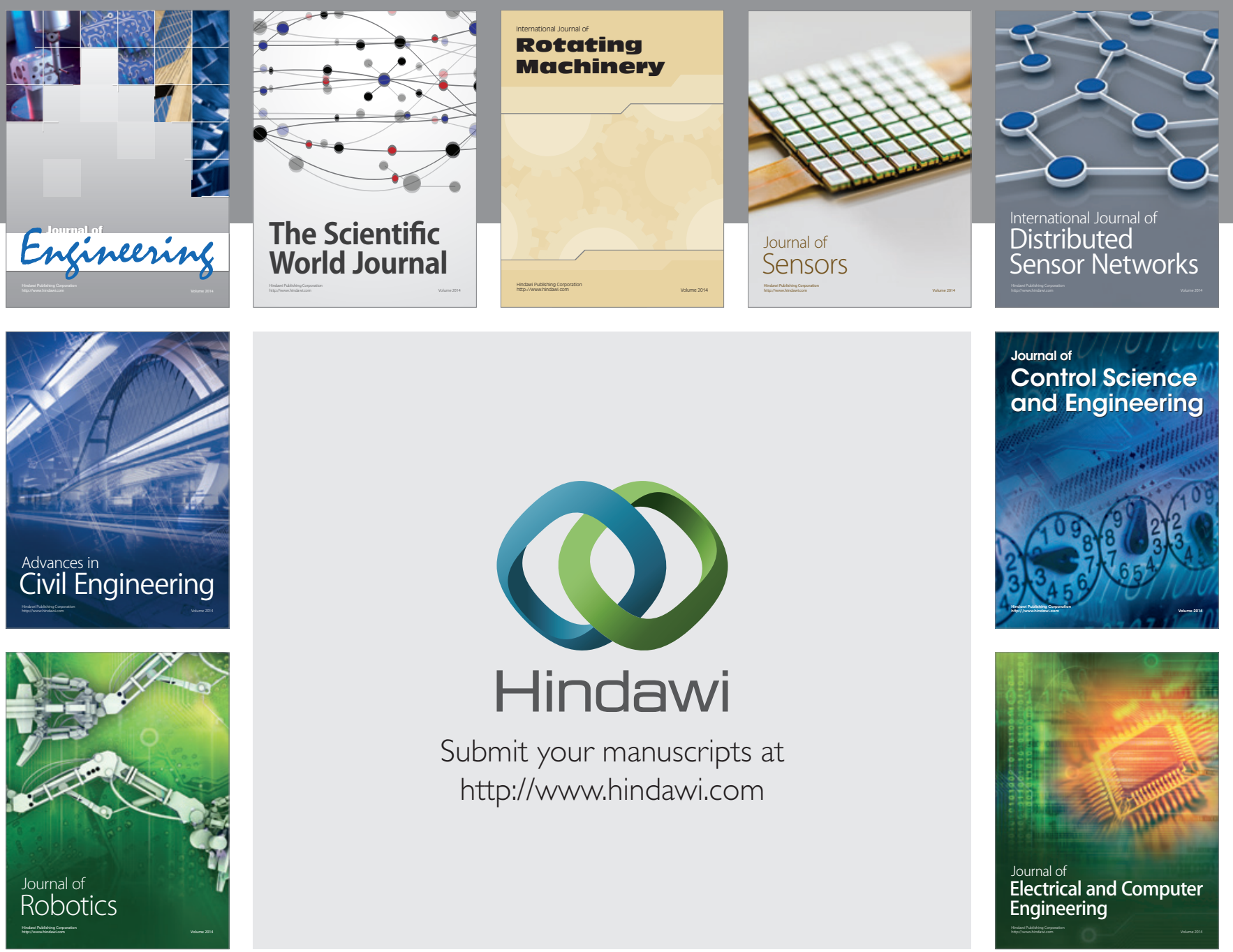

Submit your manuscripts at

http://www.hindawi.com
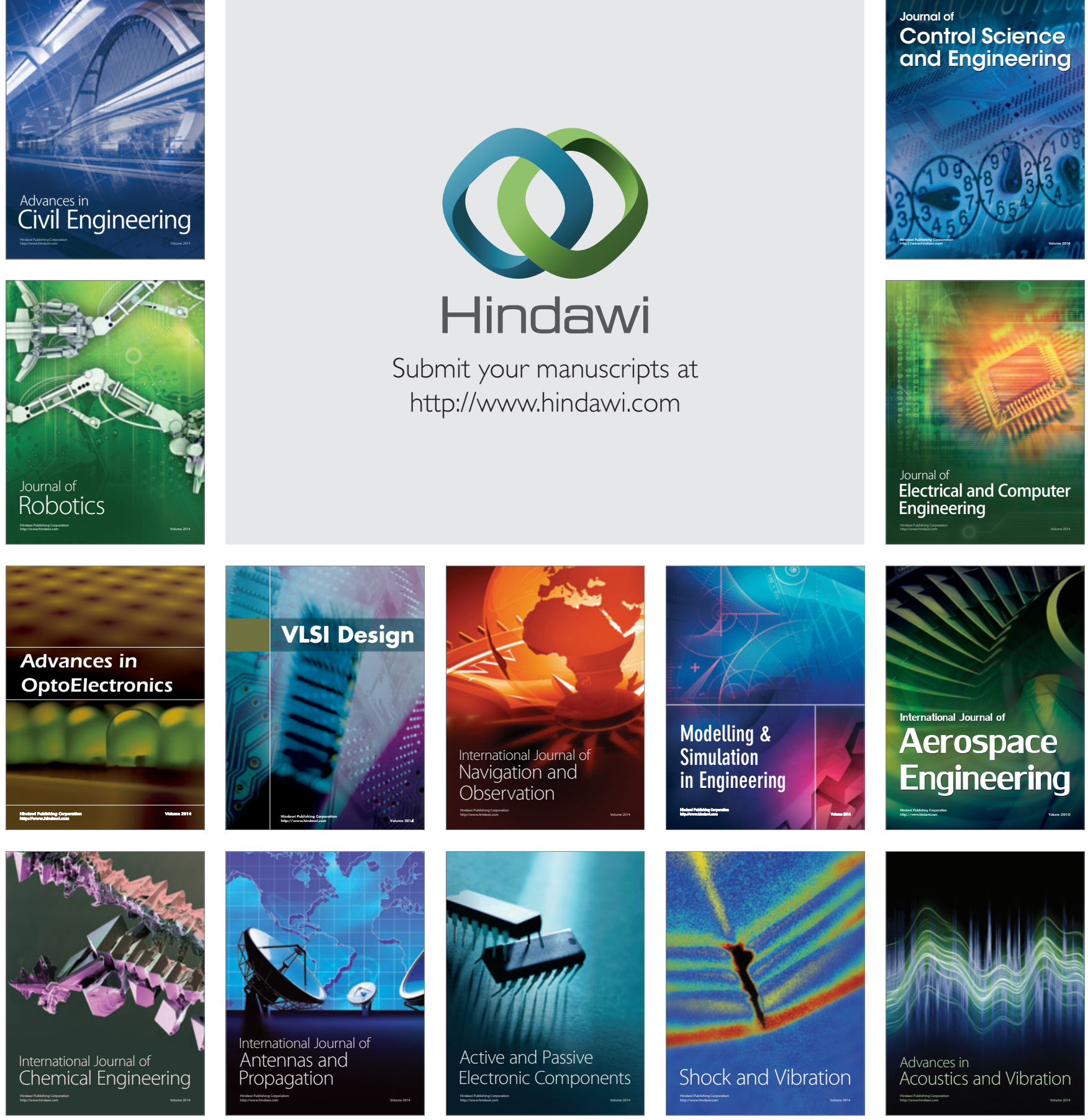